\title{
An Ontology-Driven Decision Support System for Rice Crop Production
}

\author{
Hifza Afzal ${ }^{\text {a, }}$, Mumraiz K. Kasi a ${ }^{\text {a }}$ Bakhtiar Kasi ${ }^{\text {a }}$, Bushra Naeem ${ }^{\text {a }}$, Syed Kamran Sami ${ }^{\text {b }}$ \\ ${ }^{a}$ Faculty of Information and Communication Technology, BUITEMS, Quetta \\ ${ }^{\mathrm{b}}$ Faculty of Engineering and Architecture, BUITEMS, Quetta \\ "Corresponding author's email: hifza.afzal@ buitms.edu.pk
}

\begin{abstract}
Agriculture domain now extensively uses the Internet of Things (IoTs) technology to provide farmers with proper and accurate information. Assisting farmers regularly and periodically in a more efficient manner is totally based on complete data, proper planning, and decision making. Connecting devices with each other through IoT has brought huge changes to traditional way of farming. However, it has also invited some challenges such as the semantic interoperability, quality and accuracy of data.In this paper, we extend a base farming ontology to include classes comprising of water, pesticides, and seeds information that is organized both seasonally and phase-wise. We have extended a farming ontology specifically a crop production domain using rice crop as a case study. Semantic Web Rule Language (SWRL) integrated with Jess rule engine is used for reasoning and inferencing to make devices understandable to each other. A collection of 54 SWRL rules reason about 101 OWL classes in order to maintain water irrigation in rice crops. It also provides pesticide and weedicide information for each growth stage along with seed information by identifying specific crop type. This helps the farmers to obtain better results in terms of production and sustainability from the collected data by offering them decision making support in the management of rice crops.
\end{abstract}

Keywords-Internet of Things, smart farming, semantic web, ontology, SWRL rules, Jess rule engine

Date Received: 28-09-2020

Date Accepted: 26-10-2020

Date Published: 08-06-2021

\section{INTRODUCTION}

\section{Internet of Things (IoTs) is increasingly broadening}

up its horizons to an unprecedented level, making possible the communication between the tremendous amounts of assorted devices in diverse domains. Such evolutions in IoT continuously supervene on intended consequences of growing technology, receiving favorable requirements along with a target of adverse conditions. Facing some challenges are the results of heterogeneity and complexity in devices and the data it collects, respectively [1][2]

IoT enables a variety of devices to cluster data in different formats to perform some tasks without any understanding of devices with each other due to no knowhow of description of data or information. These limitations of communication clearly reflect on domains where IoT has dominated itself. In an approach to advancement in the environment, society has embraced IoTs to define them in the form of smart cities. The idea of IoTs in smart cities improves the existing challenges to some consideration, leading to better urbanization. A growing concept of smart farming in smart cities domain influencing a future agriculture in most of the countries. Agriculture in majority countries holds an important economic value and a continuous improvement to its quality is on focus. Farmers paying particular attention to the quality of crops in time, with limited quantity of resources to get desired output in yields.

Based on an existing work [5], we emphasis on the implementation of rule engine; where, it is first important to collect and represent a complete data in the form of ontology classes. This work is extended based on farming

ontology incorporating crucial classes and a rule engine. A complete data regarding crop production is collected and then placed it in proper and adequate knowledge base integrating it with the existing OWL knowledge data. After completion of representation of data, an approach of employing rule engine is implemented. Rule engine reason and extract additional knowledge from existing entities defined.

Our work in this paper covers several prospective in a crop production domain with the help of case study in rice. We addressed the concepts missing in the ontology of farming domain. With the help of identifying some important concepts, this problem has been overcome through extending the ontology with the help of adding new classes, properties and relationships to them. Secondly, identifying the absence of rules and rule engine leads to the less efficient way of retrieving data. Adding rules assists knowledge to be reasoned and inferred with some new facts and concepts with existing knowledge. The defined rules include the rules based on irrigation management in farming, detecting polluted water source, identifying the suitable repellant, identifying a suitable seed of that specific crop. Each and every rule works according to a current growth stage of a rice crop. We employ Jess rule engine which allows to pushrules on data in order to pull desired results accordingly. This paper is organized is as follows: Section 2 presents the related work in a smart farming domain. Section 3 defines the stages and methods of our workings and provides a detailed view of base ontology and extended ontology. Section 4 presents the implementation of SWRL rules on the extended ontology. Results of inference on JESS rule engine are provided in Section 5. Finally, Section 6 concludes the work and presents future directions. 


\section{RELATED WORK}

IoT based applications on smart farming those based on Agri-IoT framework has been presented by Andreas Kamilaries et. al. [6] for the purpose to monitor two most demanding scenarios of farming 1) Heat maintenance for cow fertility 2) Finding right time for cultivation in soil fertility. Monitoring was done in real time through sensors to detect and cope up with unpredictable situations i.e Farming is highly unpredictable, due to its large dependency on weather and environmental conditions (e.g. rain, temperature, humidity, hail), unpredictable events (e.g. animal diseases, pests), as well as price volatility in agricultural markets. The solutions are then provided with the help of combined ontologies on collected static and dynamic information. The above scenarios lack online services and abilities to work on large data.

Different practices in farming have been evolved with time resulting in new ideas to lean on. Vertical farming, a new possibility has risen up showing another picture in a study of farming. Saraswati et. al. [7] have proposed an ontology based on vertical farming. Both external and internal context are taken into consideration for both monitoring and controlling the environment which in author's case is a skyscraper. The server collects the sensed data through ZigBee and updates the user at regular time intervals on GUI which is controlled via Programmable Logic Controller PLC.

Later Saraswati et. al. [8] extended their work on vertical farming ontologies aided by web services through OWL-S approach which integrates various web services. OWL-S consists of three subclasses (i.e. Service Profile, Service process and service grounding). Different concepts are taken into consideration which includes Context Based, Service Based, Environment Based, Hardware Based, and User Based concepts.

Suresh Pokharel et. al. [5] showed the conversion of heterogeneous datasets from five different domains into RDF, where datasets were linked with AGORVOC and DBpedia. The sources include Agriculture production, weather information, Crop growing days information, Soil information, Administrative data of Nepal. After conversion into ontology different quality measurements were performed on data. The author showed this process through different use cases includes 1) Irrigation In Field where crop water is calculated for some time period, 2) Agriculture planner, policy maker for planning agriculture process, 3) Agriculture Spatial Data Visualization to understand through visualized data.

\section{SMART FARMING ONTOLOGY}

\subsection{Methodology}

\subsubsection{Stages of methodology:}

Stage 1: Existing ontology defined in [5] has covered many necessary areas required in agriculture. However, we identified some important factors that has been missing which can affect the production of desired results. The additional but key factors which can influence more efficient results in a farming domain can be divided into two parts: the first part of information would be that is needed on a day-to-day basis and the second one includes information which is not often or sometimes required. The existing ontology also requires such divisions where soil type and seed type information are not required daily. Water information for agriculture is of higher priority to be perceived; as well the seeds and pesticide availability are key to manage a better farming daily plan. These classes fulfil most of the requirements regarding production of crops.

Stage 2: The extended classes along with the existing ones intend to cover a scope of crop production domain. For examining results, selecting a specific crop for results analysis to show different prospects of that domain in detail is needed. After constructing crop production ontology, rice crop is a selected as a case study for analysis of a knowledge base.

Stage 3: The addressed issues for a lack in the ontology can be efficiently gained by using a rule engine to provide reasoning through creating or reusing existing rules. SWRL Semantic web rule language is most widely used rule language, supported by protégé editor and supports many reasoners in order to combine OWL rules for semantics by proving abstract for a horn like rules. On the other side, SLOR Sensor based linked open rules is a rule discovery approach through mining algorithms having many similar traits that SWRL contains including reusability, interoperability, support for real time environments and for several reasoners lacking support for complicated sensors(e.g. GPS), distributed reasoning. According to papers [9] conclude the more emphasis on SWRL approach as it provides results without mining algorithms making it simpler and more efficient. Jess an ideal rule engine easily integrated rule engine providing rich rule-based reasoning in semantic web [10]. Creating and modifying rules in SWRL rule language and executing those rules with the help of Jess rule engine is the third step in queue.

\subsection{Ontology}

\subsubsection{Base Ontology:}

Several works have been made on ontologies for different particular domains with intentions of detailed knowledge representation for concerned objectives contenting the needs for that domain.

The existing ontology [5] containing 38 classes follow a hierarchy with respect to geographical levels from the scratch, starting from a country section, spanning an estimated scope by going forth with subclasses 'Physiography' and 'Geography'. Further, class 'Country' has been followed by a class 'Development Region'. Following the sequence, 'zone' class appears to be next in 
the list of following hierarchy. Moreover, zones in a country have furthered administratively divided into districts to separately work on different portions of distributed areas of farming.

A key classification relevant to the scope of farming is mainly based on production class which includes all the main purpose sub-classes regarding production in farming.

Agriculture within itself contains a hugely diverse area producing several productions through spanning a wide range of farming. In existing ontology, a production class covers 8 major sub-classes within the purview of concerned motives of product types including classes: Fish, Fruit, Wool, Meat, Egg, Livestock, and Milk. Besides all these classes another crucial class has also been addressed which is 'Crop production' class as a subclass of 'Production' class, producing' Crop' class, additionally also having a subclass of 'Cereal crop production' class by specifically focusing on cereal production where, it produces another class named 'Cereal' class which is also a subclass of a class 'Crop'.

Following the hierarchy 'District' class is further partitioned with class 'VDC', also 'Ward' class is a part of 'VDC' class is directed towards 'Soil' class derived from class 'Ward'. Class 'Airport' is located in class 'District'.

A weather station class named 'Station' is additionally located in 'District'. 'Station' class has three subclasses to collect different measured data from different stations. 'Rainfall Station' class collects rain relevant data from class 'Rain', where, 'Meteorology Station' as a subclass of 'Station' class collects data from 'Meteorology' class and a subclass of 'Station', the 'ETO Station' class collects data from 'Evapotranspiration' class.

\subsubsection{Extended Ontology:}

Moreover, to enhance results concluded from datasets includes some other dominating classes to infer better results. The main focus on extending an existing farming ontology is of determining and adding the missing parameters or those crucial classes which helps in obtaining more accurate, proper and unambiguous results.

Aiming to expand further the crop production specific area in the existing ontology leads to includes many important classes to make sure the completeness of this domain. However, appending those new classes that cause some adequate information lacking, includes several classes which tend to collect critical data including water, seed, and pesticide.

Taking rice as the main case study, led to work on its every growth stage separately. For the sake class 'Cereal Crop Production' is further broadened by a class 'Rice Growth Stage', shown in Figure 3.1. This class further is divided into subclasses of different phases of rice growth cycle shown in Figure 3.2.

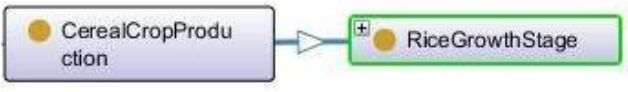

FIGURE 3.1: Class 'Rice Growth Stage'

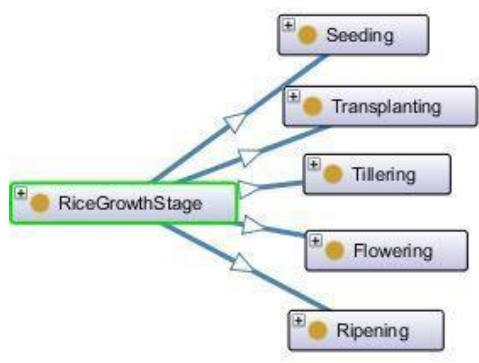

FIGURE 3.2: Class 'Rice Growth Stages' and its subclasses

A rice complete growth cycle covers some main phases, starting from the very early stage named seeding stage where seed grows on upward to the Transplanting stage. Tillering stage appears little longer after Transplanting stage as a third stage of this cycle. After these stages the rice enters into later stages of its growth cycle as Flowering phase in which it prepares it to be completely ready for the final stages. Ripening is the conclusive phase to be appeared after Flowering phase.

Another major problem that now a day's agricultural domain carries is the increasing rate of pests along with the greater number of diseases. A proper and controlled use of pesticides is of much importance not only to have control on pests but also prevent it from overuse of it that may not damage crops too.

Class 'Pesticide' and 'Weedicide' is another major class of 'Crop Production' class in Figure 3.3 and Figure 3.4 covers pesticide and weedicide area of agriculture and is a subclass of 'PestandWeed'. 'Crop production' class assists 'Pesticide' and 'Weedicide' class by identifying crop type and its growth stage.

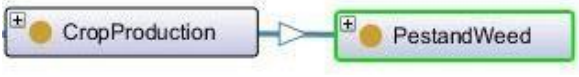

FIGURE 3.3: Class Pest and Weed

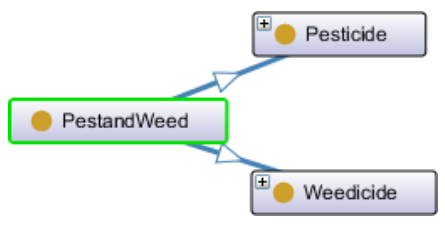

FIGURE 3.4: Class 'Pest and Weed' and its subclasses

Further, at growth stage seed class plays a vital role to keep track and then obtain different seed types information by determining a seasonal seed through crop production class in order to acquire the needed regarding information having has relation shown in Figure 3.5, where, 'Seed' has a subclass of types of seeds as 'Dicot' and 'Monocot' classes in Figure 3.6 .

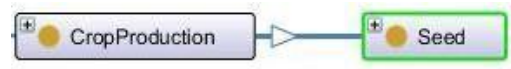

FIGURE 3.5: Class 'Seed' 


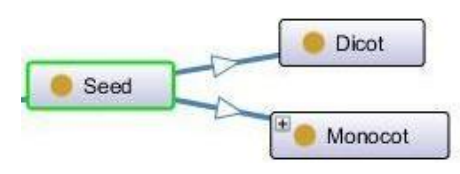

FIGURE 3.6: Class 'Seed' and its subclasses

Ward is the last in geographical hierarchy and is one of the important classes in hierarchy to be defined. Location wise Ward is the area where our case study takes place (Rice in our case) to further elaborate the required classes at this part of hierarchy in ontology. This class covers three major subclasses shown in Figure 3.7 that meet the key requisite of crop production specific domain including 'Water', 'Soil' and 'Irrigation' classes elaborated further in detail.

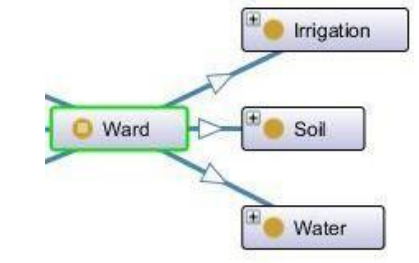

FIGURE 3.7: Class 'Ward' and its subclasses

Water, a key parameter in farming domain either for irrigation of crops or for use in livestock farming, it is always the central class. Allocation and distribution decisions of water are based on understanding and determining the water requirement both quantitatively and seasonally.

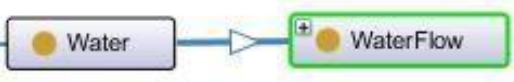

FIGURE 3.8: Class 'Waterflow'

Water class manages the schedule of water management through its subclass, the 'Water Flow' class shown in Figure 3.8, which specifies schedule of water on the basis of availability and need of water. Where, these data are collected from subclasses of this class to prevent water form overflow or drought.

To fulfill the need of required water it is necessary to know the requirement of crops. 'Water Flow' class collects that data in Figure 3.9 from its subclasses 'Very High Water Flow', 'High Water Flow', 'Moderate Water Flow', 'Low Water Flow', 'Very Low Water Flow', 'Drain Water' and 'Stop Water Flow'. Each class varies flow of water depending upon the situation. Also, each class is divided further into five subclasses except for 'Drain Water' and 'Stop Water Flow' classes shown in Figure 3.10 mapping the five-growth stage as at each growth stage water flow varies. At seeding stage 'High Water Flow' must be covering $10 \mathrm{~mm}$ of water depth, where, at flowering stage it must cover around $12 \mathrm{~mm}$.

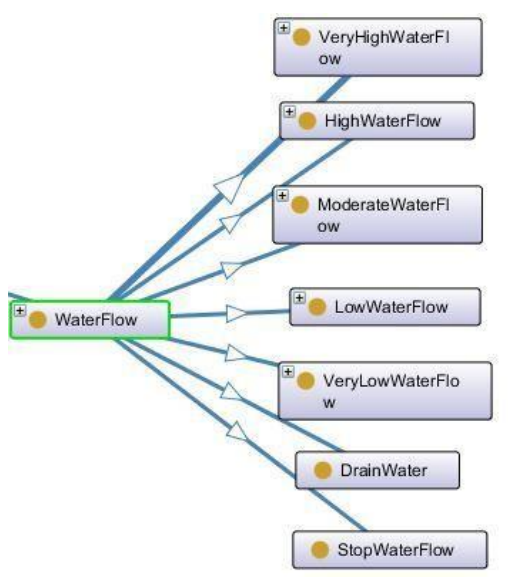

FIGURE 3.9: Class ‘Waterflow' and its subclasses

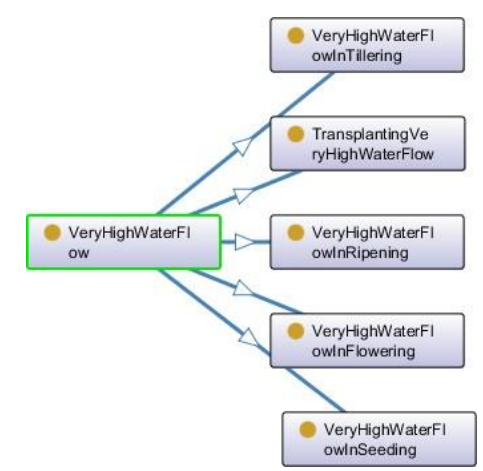

FIGURE 3.10: Class 'Very High Waterflow' and its subclasses

Taking water depth into consideration, respecting to control the water flow, it is necessary to monitor the water level at different stages of rice growth. Another subclass of 'Water' includes class 'Water Level' which observes and records the level of water and control the flow of water to its accordance shown in Figure 3.11. For example, if Seeding stage has water level greater than $10 \mathrm{~mm}$, then water flow must be controlled.

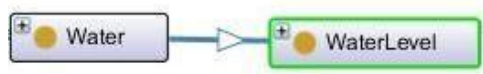

FIGURE 3.11: Class 'Water Level'

Water as a main ingredient to farming has great impact on crops. Polluted water causes not only damage to the crops but also crops cannot further be suitable for usage, even without any knowledge to the farmers. Class 'Water Measurement' a subclass of 'Water' measures the harmful nutrients in the water shown in Figure 3.12.

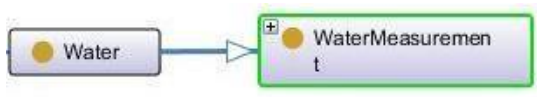

FIGURE 3.12: Class 'Water Measurement'

This class is further divided into subclasses through which it collects the data of those nutrients, including 'Magnesium Level', 'Nitrogen Level', 'Potassium Level', 'Phosphorus Level' and 'Water PH' classes. 'Polluted Water Source' is a class shown in Fig 3.13 that is the source of polluted water if occurs and is then an equivalent class of 
'Ward' to identify that specific ward containing polluted

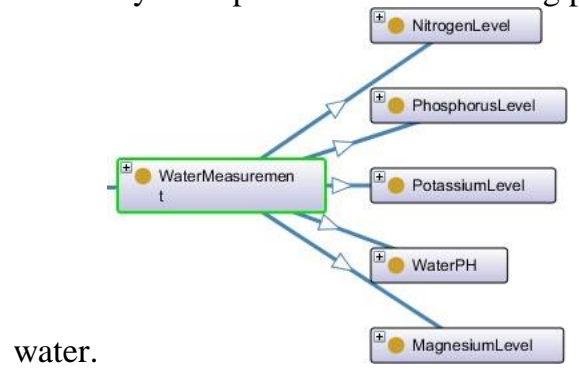

FIGURE 3.13: Class 'Water Measurement' and its subclasses

Another important subclass of class 'Ward' is class 'Irrigation'. This class collects some important data through its subclasses, class 'Season' having sub-class 'Month' and class 'Time'. Class 'Season', an extended class of 'Irrigation schedule' provides with season information along with subclass month to determine an exact duration of any season. Further, a class 'Time' provides with a specific time of day either morning, noon, evening. These classes in Figure 3.14 assist other classes and rules to know the current season and month of that season as well time of the day to provide more accuracy to the results.

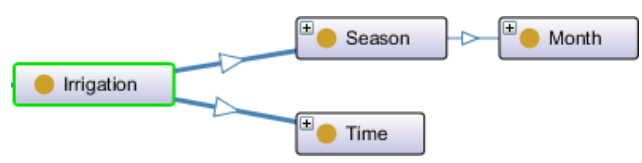

FIGURE 3.14: Class 'Irrigation' and its subclasses

The existing classes include class 'soil', an extended class of 'Ward' class in order to determine soil type holding a 'has soil' relation among. This further is subdivided into 'Soil Moisture' and 'Soil PH' classes shown in Figure 3.15. The subclass 'Soil Moisture' helps in controlling water flow if cross any threshold value, where class 'Soil PH' evaluate the quality of soil suitable for that specific crop growth.

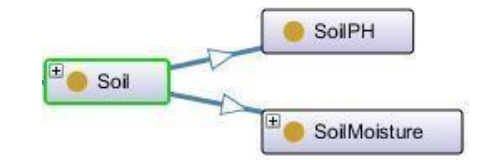

FIGURE 3.15: Class 'Soil' and its subclasses

\section{RULES IMPLEMENTATION}

Mapping those classes with each other through defining relationships between them and by default gets connected to the properties of those classes too. In order to represent the domain specific real-world data into an adequate knowledge representation to further perform reasoning on that data and infer new knowledge from those existing ones is the utmost target of this paper. This is done via implementing rule engine to infer server results through different rules. The findings of this paper, as discussed before, spins around the farming domain more specifically crop production.

After representing a complete knowledge in the form of ontology, which is the prerequisite for applying and processing rules, it is necessary to first cross check that complete graph of knowledge through any reasoner that in our case is Pellet Reasoner. This reasoner will indicate all the inconsistencies present in OWL DL (Web Ontology Language Description Logic) this also helps in SPARQL Query, execute class hierarchies and also explains the errors after inferencing.

After the required following requisites are done, the ontology is ready for implementation of rules. Rules are created and modified with the help of SWRL rule language in Protégé SWRL Tab plugin. SWRL rule contains atoms, variables, built-ins and Imp, helps in creating rules. For execution of SWRL rules it is necessary to integrate a rule engine with Protégé. Protégé previous versions support Jess rule engine, a non-open source but executes defined rules more efficiently in short time duration. Jess rule engine in Protégé 4.8.3 is used to execute SWRL based rules of our smart farming ontology.

\subsection{Decision management: The Rice crop use case}

Our proposed work provides decision making for farmers covering different parameters using Rice crop case study. The decisions and management of farming are taken geographically on a Ward. The designed method provides different defined objectives as discussed in previous sections:

1. Identifying the polluted water through sensors of that source or we can say that specific Ward that contains contaminated or polluted water

2. Identifying each growth stage in Rice crop farming to make decisions accordingly

3. Management of water during irrigation in crops in each growth stage by measuring: Soil moisture, Water level and Rainfall rate

4. Monitoring weather through Rainfall station in order to manage water during rainfall preserving the water through receiving rainfall water

5. Providing pesticide and weedicide information in each growth stage through identifying pests and weeds

6. Identifying crop in order to identify its relevant seed

As discussed previously the case study used in this paper revolves around rice crop. Our paper contains 54 SWRL rules covering most critical areas of crops to be processed. Due to brevity some of the rules and their results are defined in this paper. Before creating rules following parameters were examined after a thorough study regarding different prospects of this domain including irrigation and rainfall [13], and pesticide [11] and soil [12]. In order to index, retrieve, and organize data, AGROVOC ${ }^{1}$ provides a great range of vocabulary including 35,000 and are hierarchically organized under 25 top largely oriented towards the agricultural sector targeting mainly semantic web. Several concepts in our paper of rice are adapted from AGOVOC website, RDF-SKOS ${ }^{2}$ and AGROVOC defined Agrontology

3. The further detailed concepts and parameters about rice specific crop in crop production domain are obtained via Crop Ontology Curation Tool ${ }^{4}$. CO allows us to browse the Crop Ontology, search for specific terms in rice growth and

\footnotetext{
1 AGROVOC, http://aims.fao.org/agrovoc

${ }^{2}$ RDF-SKOS, http://aims.fao.org/agrovoc/releases

3 Agrontology, http://aims.fao.org/aos/agrontology

${ }^{4}$ Crop Ontology Curation Tool, http://www.cropontology.org/
} 
access the definition, as well as additional information. The further concepts relevant to agriculture are obtained through Chinese Agricultural Thesaurus (CAT). ${ }^{5} \mathrm{CAT}$ covers areas including agriculture, forestry, biology, etc. It is organized in 40 main categories and contains more than 63 thousand concepts. Where, the Linked Data version of CAT is in $\mathrm{RDF} / \mathrm{SKOS}-\mathrm{XL}$. In addition, CAT includes more than 130 thousand semantic relationships such as Use, UF, BT, NT and RT.

The rules are based on those perceived scenarios detailed in Table 4.1. It contains a detailed vocabulary of successful crop growth consisting concepts of cereal crop production of crop rice. Table 4.1 concepts are a corner stone for rules but also the rice ontology basic concepts and different parameters are obtained from the different papers those includes details such as soil [12], pesticide management [11], seed, activities in growth stages of rice, season, time [13] and most importantly water management [14] [15]. Where, a work based on underutilized crop production domain ontology [16] provides rules specific to Bambara groundnut providing us with different prospects of growth of crop assisting in concepts of rules making. However, the values or parameters are provided in our swrl rules are mostly obtained from different resources, developed and provided by scientists and specialists [5] [12 - 16].

Each domain in IoT expands to an unprecedented level with increasing solutions in heterogeneous devices, domains and interoperability between them. Many standardized bodies defined several ontologies that easily can be adapted to different domains with much less efforts. The official ontology we adapted in our case by looking to our requirements is Semantic Sensor Network Ontology (SOSA/SSN). SOSA ${ }^{6}$ (Sensor, Observation, Sampler and Actuator) is new extension to $\mathrm{SSN}^{7} 9$ including actuators and sampling to classes and properties. The Table 4.2 shows the extracted and analyzed requirements. The requirement column shows all of the extracted requirements and the other two parallel columns represents the covered requirements. Note that the represented requirements contain prefix followed by some identifier that might be a class or a property.

\begin{tabular}{|l|l|l|}
\hline \multicolumn{3}{|c|}{ Requirement of SOSA/SSN and Ontology } \\
\hline Requirement & SOSA/SSN & Ontology \\
\hline R1: Deployment & ssn: Deployment & $\begin{array}{l}\text { ssn: Geographical } \\
\text { level }\end{array}$ \\
\hline R2:Platform & ssn: Platform & ssn: Ward \\
\hline R3: Device & ssn: System & ssn: Device \\
\hline R3.1: Sensor & sosa: Sensor & sosa: Sensor \\
\hline R4: Actuator & sosa: Actuator & sosa: Actuator \\
\hline R5: Measurement & sosa: Observation & sosa: measurement \\
\hline R6: Property & ssn: Property & ssn: Property \\
\hline $\begin{array}{l}\text { R7: Feature of } \\
\text { interest }\end{array}$ & $\begin{array}{l}\text { sosa: Feature of } \\
\text { interest }\end{array}$ & $\begin{array}{l}\text { sosa: Feature of } \\
\text { interest }\end{array}$ \\
\hline R8: Action & sosa: Procedure & $\begin{array}{l}\text { sosa: Function, } \\
\text { command }\end{array}$ \\
\hline R9: Crop & sosa: Sample & sosa: Rice \\
\hline
\end{tabular}

TABLE 4.2: Requirement coverage by SOSA/SSN modules and Ontology

\footnotetext{
${ }^{5}$ CAT, https://bartoc.org/

${ }^{6} \mathrm{http}: / /$ www.w3.org/ns/sosa/

${ }^{7}$ http://www.w3.org/ns/ssn/
}

\subsection{SWRL Rules}

Working on details of any crop it is first important to make sure the current stage of its growth as other decisions is made on such basis. Rule 1 confirms the growth phase of rice crop. Here, RiceGrowthStage is a class having two data properties of hasStartTimeInterval indicating the start date of the Seeding phase and hasEndTimeInterval indicates the end date of the same phase with AND $(\wedge)$ operator in between implies (!) to the class Seeding as a current stage of rice growth. Water level is measured in inches per hour (in) and rainfall rate is measured as centimeter per hour $(\mathrm{cm})$. Soil moisture sensor works for low to high (wet to dry). It checks on potential of water and content of water in soil expressed as percent volumetric (1) water content. Where, the flow of water is measured as liter per minute (lpm)

RiceGrowthstage (?x)^ hasStartTimeInterval (?x, "15-May") ^ hasEndTimeInterval(?x, "15-June") $\rightarrow$ Seeding (?x)

Rule 1: Seeding Growth Stage

Following the first rule, water flow has been maintained accordingly. Soil moisture has been observed through class Soil containing data property hasSoilMoisture through fixing parameters with the help of SWRL built-ins (less than and greater than) [6]. Class Soil helps to monitor the moisture of the soil and control the water flow in accordance to the situation. Here an additional class has also been added to monitor the rainfall rate with the help of class Rainfall fixing value through the data property has value less than 5.0. This class will execute these rules till this situation has also been satisfied else will switch to the rule where rainfall rate exceeds. When all above conditions satisfy then will choose the water flow regarding that growth stage from very high to very low scale [13]. Below following rules of seeding stage are defined [15] [16]:

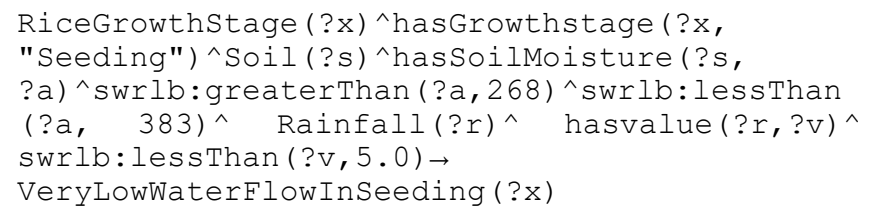

Rule 2: Very Low Water Flow

In seeding stage if and only if soil moisture is greater than 268 and less than 383 also rainfall value is less than 5.0 then Rule 2 implies very low water flow in seeding stage due to very low need of water.

RiceGrowthstage (?x)^hasGrowthstage (?x, "Seeding")^Soil (?s)^hasSoilMoisture (?s, ?a)^swrlb: greaterThan (?a, 382)^swrlb: lessThan( ?a, 418)^Rainfall (?r)^hasvalue (?r, ?v)^swrlb:le ssThan (?v, 5.0) $\rightarrow$ LowWaterFlowInSeeding (?x)

\section{Rule 3: Low Water Flow}

If soil moisture is greater than 382 and less than 418 showing less need of water, also rainfall value is less than 5.0 then Rule 3 implies to allow low water flow in seeding stage. 


\begin{tabular}{|l|l|}
\hline \multicolumn{2}{|c|}{ Rice Crop Vocabulary } \\
\hline Class & Cereal Crop Production \\
\hline Instance & Rice \\
\hline Superclass & Cop Production \\
\hline Soil & Loam \\
\hline Soil Moisture & Varies in Different growth stages \\
\hline Soil PH & Greater than 5.0 and less than 6.5 \\
\hline Rainfall & Suitable rainfall rate ( less than 0.5) \\
\hline Water Level & In millimeters \\
\hline Polluted Water & If greater than 1.5 \\
\hline Pesticide & Carbaryl, Nateevo \\
\hline Weedicide & Sunstar \\
\hline Seed name & Orziya Sativa \\
\hline Season & Summer, Spring, Winter, Autumn \\
\hline Growth stage & $\begin{array}{l}\text { Seeding, Transplanting, Tillering, Flowering, } \\
\text { Ripening, Harvesting }\end{array}$ \\
\hline Growth Information & $\begin{array}{l}\text { Rice crop growth depends on soil type (Loam) and on soil moisture (dry, normal, wet), a suitable } \\
\text { soil moisture on suitable growth stage is necessary. Soil PH with suitable temperature and } \\
\text { moderate rainfall rate or controlled water rate maintains a successful growth and good yield. Water } \\
\text { flow completely depends in a soil moisture, rainfall rate and water level. Polluted water is not } \\
\text { tolerated as it may leads to damage to the crops (Magnesium, Phosphorus, Nitrogen, Potassium } \\
\text { and water PH levels must be less than 1.5). At early stages of the growth pesticide use can be } \\
\text { harmful that's why a weedicide (Sunstar) is suitable for those early stages and pesticide (nateevo) } \\
\text { at the middle stages of the growth. When crops enters into maturity phases } \\
\text { pesticide (carbaryl) can be used to prevent it from pests. }\end{array}$ \\
\hline
\end{tabular}

TABLE 4.1: Rice Crop Vocabulary

RiceGrowthStage (?x)^hasGrowthstage (?x, "Seeding")^Soil(?s)^hasSoilMoisture(?s, ?a)^swrlb: greaterThan (?a,417)^swrlb: lessThan ( ?a, 435)^Rainfall (?r)^hasvalue (?r, ?v)^ swrlb: lessThan (?v, 5.0) $\rightarrow$

ModerateWaterFlowInSeeding (?x)

\section{Rule 4: Moderate Water Flow}

If soil moisture is greater than 417 and less than 435 indicating normal moisture of soil, also rainfall value is less than 5.0 then Rule 4 implies to allow moderate water flow in seeding stage.

RiceGrowthStage (?x)^hasGrowthstage (?x, "Seeding")^Soil(?s)^hasSoilMoisture(?s, ?a)^swrlb: greaterThan (?a,434)^swrlb: lessThan( ?a, 451)^Rainfall (?r)^hasvalue (?r, ?v)^swrlb:le ssThan $(? \mathrm{~V}, 5.0) \rightarrow$ HighWaterFlowInSeeding $(? \mathrm{x})$

Rule 5: High Water Flow

In seeding stage if and only if soil moisture is greater than 434 and less than 451 indicating dry soil, also rainfall value is less than 5.0 then Rule 5 implies the need of high water flow.

RiceGrowthStage (?x)^hasGrowthstage(?x, "Seeding")^Soil(?s)^hasSoilMoisture (?s, ?a)^swrlb:e qual (?a, 1023)^Rainfall(?r)^hasvalue(?r, ?v) ^ swrlb: lessThan (?v, 5.0) $\rightarrow$

VeryHighwaterFlowInSeeding (?x)

\section{Rule 6: Very High Water Flow}

In seeding stage if and only if soil moisture is equal to 1023 showing completely dry, also rainfall value is less than 5.0 then Rule 6 implies the need very high water flow in seeding stage. In case of high rainfall, the above rules will not be executed as the Rainfall condition becomes false. This will not only help to save water and utilize from the rain water but also prevent crops from flooding.

Rainfall (?r)^hasvalue (?r, ?v)^swrlb:greaterTha $\mathrm{n}(? \mathrm{v}, 5.0) \rightarrow$ StopWaterFlow (?r)

\section{Rule 7: Stop Water Flow}

As soon as rainfall rate exceed 5.0 values it will stop the water flow shown in Rule 7.

RiceGrowthStage (?x)^ hasGrowthstage (?x, "Seedin g")^Soil (?s)^hasSoilMoisture (?s, ?a)^swrlb:gre aterThan (?a, 417) ^ swrlb:lessThan $(? a, 435) \wedge$ WaterLevel (?w) ^ hasWaterLevel (?w, ?y) ^ swrlb:lessthan(?y, 2) ^ Rainfall(?r) ^ hasvalue(?r, ?v) ^ swrlb:lessThan(?v, 5.0) $\rightarrow$ ModerateWaterFlowInSeeding ( ?x)

Rule 8: Moderate Water Flow

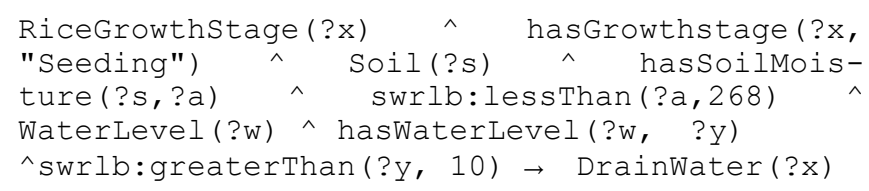

Rule 9: Drain Water

Class WaterLevel in Rule 8 observes the level of water in that specific phase of growth, if water reaches below threshold then starts to flow the moderate flow of water or if exceeds the water level in Rule 9 due to rainfall then drain the extra water [13]. In almost each rule, growth stage has been observed first as every stage of growth has different requirements.

It is always not necessary that crops get fresh supply of water from either source [14] or it is easy to estimate it through the surface. The following upcoming rules assist to 
measure increasing rates of the different water pollutant nutrients. If any of the rules satisfies the condition it will indicate the red flag of polluted water source which is also equivalent to that specific polluted ward. Five different harmful nutrients have been measured with the help of data property hasvalue and SWRL built-in (greater than).

Ward (?x)^hasWard (?x, "Ward") ^MagnesiumLevel (?a ) ^ hasvalue(?a, ?b) ^ swrlb:greaterThan(?b, $1.0) \rightarrow$ PollutedWaterSource(?x)

Rule 10: Magnesium Polluted Water

Rule 10 implies polluted water source if magnesium level is greater than 1.5 [15].

Ward(?x) ^ hasWard(?x, "Ward") ^ WaterPH(?c)

$\wedge$ hasvalue(?c, ?d) ^ swrlb:greaterThan(?d,

1.5) $\rightarrow$ PollutedWaterSource(?x)

Rule 11: Water PH Polluted Water

Rule 11 implies polluted water source if water $\mathrm{PH}$ is greater than 1.5.

Ward (?x)^hasWard (?x, "Ward")^NitrogenLevel (?e) ^hasvalue (?e, ?f)^swrlb:greaterThan(?f, 1.5) $\rightarrow$ PollutedWaterSource (?x)

Rule 12: Nitrogen Polluted Water

If nitrogen level exceeds value 1.5 Rule 12 implies polluted water source.

Ward (?x)^hasWard ( ?x, "Ward")^PotassiumLevel (?g )^hasvalue(?g, ?h)^swrlb:greaterThan(?h, 1.5)

$\rightarrow$ PollutedWaterSource (?x)

Rule 13: Potassium Polluted Water

Rule 13 implies polluted water source if potassium level is greater than 1.5 .

Ward (?x)^hasWard (?x, "Ward")^PhosphorusLevel (? i) ^ hasvalue(?i, ?j) ^ swrlb:greaterThan(?j, 1.5) $\rightarrow$ PollutedWaterSource (?x)

\section{Rule 14: Phosphorus Polluted Water}

If phosphorus level is greater than value 1.5 Rule 14 implies polluted water source.

Pesticide and weedicide are two different repellents used in different growing stages of crops if spotted. The following rules implicate the suitable repellent with respect to every growing phase [15] [25].

RiceGrowthStage (?x)^hasGrowthstage (?x, "Tiller ing") ^ Pesticide(?r) ^ hasvalue(?r, ?v) ^ swrlb: greaterThan(?v, 0.5) $\rightarrow$ Nateevo(?x)

Rule 15: Nateevo Pesticide

If stage tillering detect pesticide and has value greater than value 0.5 , then Rule 15 implies nateevo pesticide.

RiceGrowthstage (?x)^hasGrowthstage (?x,

"Seeding")^ Weedicide(?r) ^ hasvalue(?r, ?v)

$\wedge$ swrlb:greaterThan(?v, 0.5) $\rightarrow$ Sunstar(?x)

Rule 16: Sunstar Weedicide

Rule 16 implies sunstar weedicide if weedicide has value greater than 0.5 in seeding stage.

RiceGrowthstage (?x)^hasGrowthstage (?x,

"Ripening")^Pesticide(?r) ^ has-value(?r, ?v)

$\wedge$ swrlb:greaterThan(?v, 0.5) $\rightarrow$ Carbaryl(?x)

Rule 17: Carbaryl Pesticide
Rule 17 implies carbaryl pesticide if pesticide value is greater than 0.5 in ripening stage.

RiceGrowthStage ( ?x) ^hasGrowthstage (?x, "Flowering")^Pesticide(?r)^ hasvalue(?r, ?v)

$\wedge$ swrlb:greaterThan(?v, 0.5) $\rightarrow$ Carbaryl(?x)

Rule 18: Carbaryl Pesticide

If stage flowering detect pesticide and has value greater than value 0.5 , then Rule 18 implies carbaryl pesticide.

RiceGrowthStage (?x)^hasGrowthstage (?x, "Transplanting")^Weedicide(?r)^ hasvalue(?r, ?v)^swrlb:greaterThan(?v, 0.5) $\rightarrow$ Sunstar(?x)

Rule 19: Sunstar Weedicide

If stage transplanting detects pesticide and has value greater than value 0.5 , then Rule 19 implies sunstar weedicide.

Crop(?c)^hasCrop(?c,"Rice") $\rightarrow$ OryzaSativaSeed(?c)

Rule 20: Oryza Sativa Seed

Where, a seed with respect to that specific crop is identified if that crop occurs in the following Rule 20.

\section{RESULTS}

\subsection{Results}

Before the final execution each rule was executed separately to test all of the rules according to their values. As soon as all results were found satisfying the final analysis on all of the rules were made.

One of the major benefits of using Jess rule engine is its very quick response for executing results. It takes milliseconds to convert SWL rules into Owl Knowledge, executing the rules in the jess rule engine and again transferring inferred results to Owl knowledge. This swift process makes it an efficient way of getting valid results in no time.

\subsubsection{Seeding growth stage rules:}

\section{a) Response time:}

For executing seeding stage rules the estimated response time are shown in Figure 5.1 below. Converting SWRL rules and OWL knowledge by converting OWL and SWRL into Jess engine, the calculated running time is 407 milliseconds. In this time bound it processed 8 imported SWRL rules, 101 classes, 17 individuals and collectively imported 125 axioms to the rule engine.

\section{$\rightarrow$ SMRLJessBridge $\rightarrow$ Rules $\rightarrow$ Classes $\rightarrow$ Individuals $\rightarrow$ Axioms $\rightarrow$ Inferred Axiom}

SMRL rule and relevart $O M L$ knowledge successfully converted to rule engine knowledge.

Number of SMRL rules exported to rule engine: 8

Number of OML class declarations exported to rule engine: 101

Number of $\mathrm{OM}$ individual declarations exported to rule engine: 17

Number of other OM axioms exported to rule engine: 125

The transfer took 407 millisecond(s).

Press the "Run Jess" button to run the rule engine.

FIGURE 5.1: Response time of converting OWL and SWRL into Jess

Executing all seeding stage specific Jess rules, it took 46 milliseconds to infer 3 axioms in Figure 5.2.

$\rightarrow$ SMRLJessBridge $\rightarrow$ Rules $\rightarrow$ Classes $\rightarrow$ Individuals $\rightarrow$ Axioms $\rightarrow$ Inferred Axiom

Successful execution of rule engine

Number of inferred axioms: 3

The process took 46 millisecond(s).

Look at the "Inferred Axioms" tab to see the inferred axioms.

Press the "Jess- $\rightarrow$ OML" button to translate the asserted facts to OM knowledge. 
FIGURE 5.2: Response time of executing seed rules

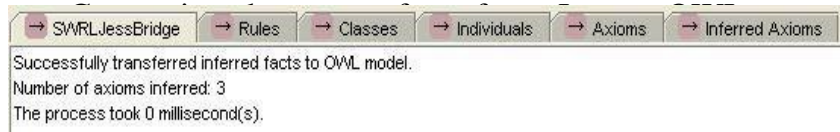

FIGURE 5.3: Response time of transferring facts to OWL model

After compiling Seeding stage based Jess rules the two inferred results become as the output includes: (a) 'Seeding' as a current growth stage of rice crop based on the time intervals of this stage (b) 'ModerateWaterFlow' class after fulfilling the condition of the need of more water to the crops based on low soil moisture and low rate of rainfall. model took almost no time or it can just be a 0.5 , as shown in Figure 5.3.

In case of different values of soil moistures, the different water flow rates become the inferred results either allowing for flow of water or controlling it.

In case of low water level and low soil moisture showing need of water results in inferred axioms showing two classes of 'ModerateWaterFlow' in Figure 5.4. The first one class is the result from rule of low soil moisture and low rainfall rate and the second one is the result of low soil moisture and low water level in seeding stage.

\section{$\rightarrow$ SMRLJessBridge $\rightarrow$ Rules $\rightarrow$ Classes $\rightarrow$ Individuals $\rightarrow$ Axioms $\rightarrow$ Inferred Axioms

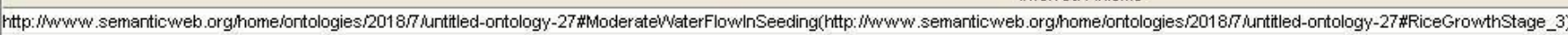

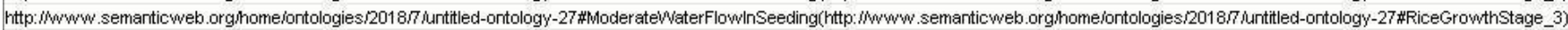 http:/MWw semanticweb.org/home/ontologies/2018//untitled-ontology-27\#Seeding(http:/WwW. semanticweb.orghome/ontologies/2018//untitled-ontology-27 \#RiceGrowthStage_3) \\ FIGURE 5.4: Moderate water flow in seeding stage}

If a water level and a specific soil moisture value exceed the threshold then 'DrainWater' class becomes an output to drain water till it reaches to a suitable water level and soil moisture value shown in Figure 5.5.

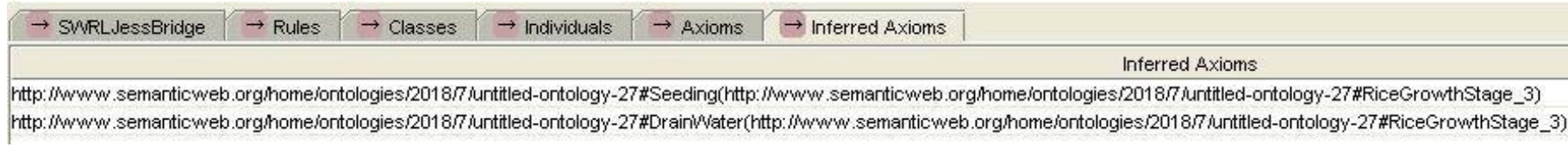

FIGURE 5.5: Drain water in seeding stage

\section{CONCLUSION AND FUTURE WORK}

Semantic web technologies assist IoT based domains by allowing IoT devices to access heterogeneous data from network, presented in the form of RDF representation in ontology. Sematic web is used in many areas of agriculture, where, the accurate data and proper decision making is of utmost importance to the farmers. In this work, we have added non-existing classes mainly of water, pesticide and seeds in crop specific production with the help of rice crop as a test case study. The results obtained were inferred through Jess rule engine to conclude new facts through reasoning knowledge-based data. Implementation of rule engine provides a way to collect and process huge data in the form of ontology and make complex decisions without human intervention in real time. Our ontology contains a set of 54 rules and 101 classes.

A lacking prosperity of agriculture results in declining of field management. The rules we generated from existing and newly added classes covers a proper water management in different scenarios of basmati rice. Also, pesticide management and seed information are maintained. The successful results drawn covered different stages of rice growth of rice. This helps crop knowledge management and improves quality of services in crop farming.

Agriculture with in itself is a wide area containing a lot several domains. There is ample room for further enhancement in ontology and enhancement to the rule base. Improvements in ontology can be done in any stage of ontology lifecycle. The workings of this paper cover many crucial classes to infer new facts in crop specific domain. This single scope extended ontology retrieved results of different parameters. Beside all those classes the proposed results from smart farming ontology can be elaborated adding some other additional classes that can ease farmers in almost every way including fertilizer, detecting diseases and new agricultural equipment details. Apart from crop production domain, several other farming domains are uncovered in this paper. Hence, this paper attempt to devise new approaches is belonging to other farming concepts including fish, meat, egg, fruit, wool, livestock, milk farming. Further it can be extended for other farming domains like vegetable farming, poultry farming and spice farming. 


\section{REFERENCES}

[1] A. Lawan, A. Rakib, N. Alechina, and A. Karunaratne, "Advancing underutilized crops knowledge using swrl-enabled ontologies-a survey and early experiment." in JIST (Workshops \& Posters), 2014, pp. 69-84.

[2] A. I. Maarala, X. Su, and J. Riekki, "Semantic reasoning for context aware internet of things applications," arXiv preprint arXiv:1604.08340, 2016.

[3] M. Grobe, "Rdf, jena, sparql and the'semantic web'," in Proceedings of the 37th annual ACM SIGUCCS fall conference: communication and collaboration. ACM, 2009, pp. 131-138.

[4] A. Kamilaris, F. Gao, F. X. Prenafeta Boldú, and M. I. Ali, "Agri- iot: A semantic framework for internet of things enabled smart farming applications," in Internet of Things (WF-IoT), 2016 IEEE 3rd World Forum on. IEEE, 2016, pp. 442-447.

[5] S. Pokharel, M. A. Sherif, and J. Lehmann, "Ontology based data access and integration for improving the effectiveness of farming in nepal," in Proceedings of the 2014 IEEE/WIC/ACM International Joint Conferences on Web Intelligence (WI) and Intelligent Agent Technologies (IAT)Volume 02. IEEE Computer Society, 2014, pp. 319-326.

[6] A. Jemal, H. Ktait, R. B. Halima, and M. Jmaiel, "Oodaas: Ontology driven analysis for self adaptive ambient systems," in Proceedings of the International Conference on Internet of things and Cloud Computing. ACM, 2016, p. 66.

[7] S. Sivamani, N. Bae, and Y. Cho, "A smart service model based on ubiquitous sensor networks using vertical farm ontology," International Journal of Distributed Sensor Networks, vol. 9, no. 12, p. 161495 , 2013.

[8] S. Sivamani, H.-g. Kim, M. Lee, J. Park, C. Shin, and Y. Cho, "An ontology model for smart service in vertical farms-an owl-s approach," International Journal of uand eService, Science and Technology, vol. 9, no. 1, pp. 161-170, 2016.
[9] M. O'connor, H. Knublauch, S. Tu, and M. Musen, "Writing rules for the semantic web using swrl and jess," Protégé With Rules WS, Madrid, 2005.

[10] A. Gyrard, M. Serrano, J. B. Jares, S. K. Datta, and M. I. Ali, "Sensor-based linked open rules (slor): An automated rule discovery approach for iot applications and its use in smart cities," in Proceedings of the 26th International Conference on World Wide Web Companion. International World Wide Web Conferences Steering Committee, 2017, pp. 11531159.

[11] S. Arora, I. Mukherji, A. Kumar, and R. Tanwar, "Pesticide residue analysis of soil, water, and grain of ipm basmati rice," Environmental monitoring and assessment, vol. 186, no. 12, pp. 8765-8772, 2014.

[12] G. Gines, J. Bea, and T. Palaoag, "Characterization of soil moisture level for rice and maize crops using gsm shield and arduino microcontroller," in IOP Conference Series: Materials Science and Engineering, vol. 325, no. 1. IOP Publishing, 2018, p. 012019.

[13] D. Wang and X. Cai, "Irrigation scheduling - role of weather forecasting and farmers' behavior," Journal of water resources planning and management, vol. 135, no. 5, pp. 364-372, 2009.

[14] J. Mateo-Sagasta, S. M. Zadeh, H. Turral, and J. Burke, Water pollution from agriculture: a global review. Executive summary. Rome, Italy: FAO Colombo, Sri Lanka: International Water Management Institute (IWMI). CGIAR Research Program on Water, Land and Ecosystems (WLE)., 2017.

[15] M. P. Polson and S. Eswaran, "Ontology creation and semantic web for paddy," International Journal of Computer applications, vol. 41, no. 9, 2012.

[16] A. Thunkijjanukij, A. Kawtrakul, S. Panichsakpatana, U. Veesommai et al., "Ontology development: a case study for thai rice," Kasetsart J.(Nat. Sci.), vol. 43, no. 3, pp. 594-604, 2009.

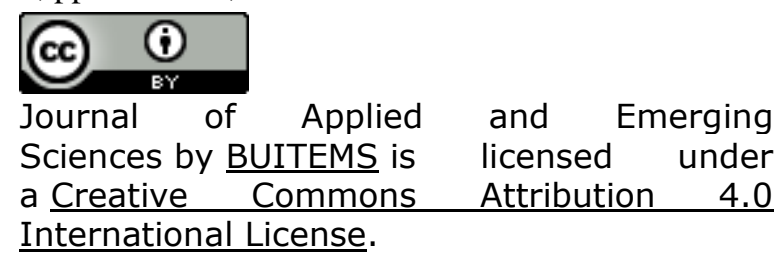

